\title{
DIFUSÃO DE CONTAMINANTES EM CONDIÇÕES DE VENTO FRACO EMPREGANDO UM MODELO ESTOCÁSTICO LAGRANGEANO
}

\author{
ANDRÉA UCKER TIMM ${ }^{1}$, DÉBORA REGINA ROBERTI ${ }^{2}$, GERVÁSIO ANNES DEGRAZIA ${ }^{2}$ E \\ DOMENICO ANFOSSI ${ }^{3}$ \\ ${ }^{1}$ Pós-Graduação em Física. UFSM/LuMet, Santa Maria, RS, Brasil. \\ ${ }^{2}$ Departamento de Física/UFSM, Santa Maria, RS, Brasil. \\ ${ }^{3}$ C.N.R., Istituto di Scienze dell'Atmosfera e del Clima, Torino, Italy. \\ annddri@yahoo.com.br,d_r_roberti@yahoo.com.br \\ degrazia@ccne.ufsm.br, anfossi@isac.cnr.it
}

Recebido Julho 2007 - Aceito Março 2009

\begin{abstract}
RESUMO
Um modelo estocástico Lagrangeano é utilizado para simular a dispersão e o transporte de contaminantes, sob condições estáveis, com velocidade do vento fraco, no experimento traçante realizado no Idaho National Engineering Laboratory (INEL). Neste trabalho é testada uma nova parametrização dos parâmetros que representam as freqüências associadas ao fenômeno de meandro do vento, presentes nas equações de Langevin para as componentes do vento horizontal. Esta nova parametrização é descrita em termos de uma quantidade adimensional que controla a freqüência de oscilação de meandro do vento e da escala de tempo, associada às estruturas coerentes em uma turbulência bem desenvolvida. As simulações demonstram que o modelo Lagrangeano considerado, incorporando esta nova parametrização, reproduz corretamente a difusão de escalares passivos em uma camada limite atmosférica estável com velocidade do vento fraco.
\end{abstract}

Palavras-chave: velocidade do vento fraco, meandro do vento, dispersão.

\begin{abstract}
DIFUSION OF CONTAMINANTS IN LOW WIND CONDITIONS EMPLOYING A LAGRANGIAN STOCHASTIC MODEL

A Lagrangian stochastic particle model is utilized to simulate the dispersion and the transport of contaminants under low wind stable conditions in the tracer experiment carried in the Idaho National Engineering Laboratory (INEL). In this work a new parameterization for the parameters representing the frequencies associated to the meandering phenomenon is tested. The new parameterization is expressed in terms of a non-dimensional quantity that controls the frequencies of the meandering oscillation and of the time scale associated to a coherent structure in a fully developed turbulence. The simulations show that the considered Lagrangian model, incorporating this new parameterization, reproduces correctly the diffusion of passive scalars in a low wind speed stable atmospheric boundary layer.
\end{abstract}

Keywords: Low wind speed, meandering, dispersion. 


\section{INTRODUÇÃO}

Nas últimas décadas, a dispersão de poluentes na atmosfera tornou-se um assunto bastante estudado, e a importância do tema tornou-se ainda maior com o aumento do número de emissões de contaminantes na atmosfera. Um fator decisivo, que tem motivado o estudo detalhado do transporte $\mathrm{e}$ da dispersão de poluentes na atmosfera, é a exigência de uma geração sempre maior de energia em sociedades modernas, que desenvolvem inovações tecnológicas e apresentam uma rápida industrialização. Concomitantemente, o aumento da população em áreas primitivamente ocupadas por florestas, fez com que a poluição do ar representasse um grande problema para países em desenvolvimento.

Analisando-se os argumentos acima, se conclui que a determinação do transporte de poluentes na atmosfera é um fator importante no gerenciamento ambiental, envolvendo o conhecimento detalhado da evolução temporal e espacial da Camada Limite Planetária (CLP). Face às dificuldades existentes, principalmente associadas aos altos custos, na caracterização observacional através de campanhas de medidas, o transporte de poluentes na atmosfera é normalmente avaliado através de modelos matemáticos.

Os modelos matemáticos que determinam as propriedades da atmosfera e os seus efeitos sobre os poluentes são denominados modelos de dispersão, os quais representam uma importante ferramenta de investigação dos transportes de poluentes na atmosfera. Devido à estrutura complexa da CLP, onde os campos de vento e a turbulência são não-homogêneos e não-estacionários, a aplicação destes modelos para a dispersão de poluentes é uma tarefa que encontra inúmeras dificuldades. A aplicação de modelos de partículas estocásticos Lagrangeanos tem sido normalmente aconselhada, devido à natureza Lagrangeana do movimento das partículas, uma vez que elas se movem seguindo o escoamento.

Devido ao bom entendimento da estrutura turbulenta da CLP durante condições de ventos fortes, a dispersão de poluentes sobre estas condições tem sido modelada satisfatoriamente. Por outro lado, em situações de ventos fracos e especialmente durante condições estáveis, a estrutura turbulenta da CLP é deficientemente conhecida (Sharan et al., 1995). Como resultado, a ocorrência de velocidades do vento fraco é geralmente considerada a situação mais crítica para a dispersão de poluentes em distâncias próximas e médias da fonte de emissão (Cirillo e Poli, 1992). A difusão de poluentes liberados sob tais condições é considerada irregular e indefinida, pois a pluma de contaminantes fica sujeita a grandes oscilações horizontais do vento. Na literatura, estas oscilações do vento horizontal são chamadas de meandro, e são responsáveis pelo fato das funções de autocorrelação observadas para as componentes do vento horizontal, exibirem um comportamento oscilatório, caracterizado pela presença de grandes lóbulos negativos (Anfossi et al., 2005a e Oettl et al., 2005).

De acordo com Oettl et al. (2005) esta oscilação do vento horizontal de baixa freqüência é um fenômeno relacionado às estruturas das equações de Navier-Stokes. Ela resulta de uma condição física especial, em que o equilíbrio entre a força de Coriolis e o gradiente de pressão gera uma solução que exibe comportamento oscilatório. Apesar do fenômeno de meandro do vento parecer existir na maioria das condições de estabilidade, seu efeito sobre a dispersão de contaminantes é mais evidente durante condições estáveis com a presença de ventos fracos, onde não prevalecem os efeitos de uma turbulência bem desenvolvida.

Neste sentido, o objetivo deste trabalho é utilizar o modelo de dispersão estocástico Lagrangeano LAMBDA (Brusasca et al., 1992; Ferrero e Anfossi, 1998 a,b) para simular a dispersão e o transporte de poluentes em condições estáveis com velocidade do vento fraco e terreno plano. $\mathrm{O}$ experimento traçante realizado no Idaho National Engineering Laboratory (INEL) foi considerado. A principal contribuição deste trabalho é testar uma nova parametrização dos parâmetros e , que representam as freqüências associadas ao fenômeno de meandro do vento, presentes nas equações de Langevin para as componentes do vento horizontal. Esta nova parametrização é descrita em termos dos valores de e , onde é uma quantidade adimensional que controla a freqüência de oscilação do meandro, e é a escala de tempo associada às estruturas coerentes em uma turbulência bem desenvolvida. Esse conjunto de equações de Langevin para as componentes horizontais do vento foi testado recentemente por Anfossi et al. (2006). Eles calcularam os valores de e ajustando a equação para a função de autocorrelação Euleriana, obtida a partir de dados experimentais, estimando diferentes pares de e para cada experimento e para cada altura da torre $(2,4,8,16,32 \mathrm{e}$ $61 \mathrm{~m}$ ), enquanto aqui será apresentada uma nova parametrização para essas variáveis físicas.

\section{METODOLOGIA}

\subsection{Formulação matemática para modular a dispersão em situações de vento fraco}

Simular a dispersão de escalares passivos em condições de vento fraco é um problema físico difícil, pois quando a velocidade do vento decresce abaixo de certo valor crítico $\left(\bar{u}<1.5 \mathrm{~ms}^{-1}\right)$, não é possível definir uma direção precisa do vento médio (Oettl et al., 2005). Assim, tornando-se crucial estimar corretamente a dispersão de poluentes na atmosfera. Neste contexto, foi proposto por Murgatroyd (1969) a seguinte relação, 


$$
R(\tau)=e^{-p \tau} \cos (q \tau)
$$

e a mesma expressão com

$$
p=\frac{1}{\left(m^{2}+1\right) T} \quad \text { e } \quad q=\frac{m}{\left(m^{2}+1\right) T}
$$

foi sugerido por Frenkiel (1953). Esta forma funcional é composta do produto da função exponencial clássica (representado pela função de autocorrelação para uma turbulência bem desenvolvida) pela função cosseno (a qual descreve o fenômeno de meandro associado às oscilações de baixa freqüência do vento horizontal). As Equações 1a e $1 \mathrm{~b}$ contêm os parâmetros $p$ e $q$. As grandezas $p$ e $q$ constituem parâmetros híbridos expressos em termos de $T$, que representa uma escala de tempo associada a uma turbulência bem desenvolvida, e $m$ uma quantidade sem dimensão que controla a freqüência de oscilação do fenômeno de meandro ou o seu período. $\mathrm{O}$ parâmetro $m$ controla o valor absoluto do lóbulo negativo na função de autocorrelação Euleriana, e estabelece a magnitude do fenômeno de meandro. A formulação dada pela função (1a) reproduz a função de autocorrelação Euleriana $\mathrm{R}(\tau)$ experimental em situações de velocidade do vento fraca (Anfossi et al., 2005a). A forma exponencial clássica quando aplicada a casos de meandro, não reproduz o comportamento observado das funções de autocorrelação (Oettl et al., 2005). Em particular, a forma de Frenkiel recupera o resultado clássicom, quando os efeitos de meandro não são considerados, ou seja, quando $m=0$. Desta forma, pode-se dizer, que do ponto de vista observacional, a fórmula algébrica de Frenkiel expressa pela Equação 1a apresenta uma flexibilidade empírica.

Anfossi et al. (2005a) e Oettl et al. (2005) derivaram um novo sistema de equações de Langevin acopladas para as componentes horizontais do vento. Esse sistema de equações foi proposto para simular a dispersão em condições de vento fraco, condições homogêneas e estacionárias:

$$
d u=-(p u+q v) d t+\sigma_{u} \sqrt{2 p d t} \xi u
$$

e

$$
d v=-(-q u+p v) d t+\sigma_{v} \sqrt{2 p d t} \xi v
$$

onde $u$ e $v$ são as componentes horizontais das flutuações da velocidade do vento, $\xi u$ e $\xi v$ são variáveis Gaussianas (tendo média zero e variância $d t$ ), $\sigma_{\mathrm{u}}$ e $\sigma_{\mathrm{v}}$ são os desvios padrões das componentes horizontais do vento.

O segundo termo na Equação 2, o qual representa a difusão, foi obtido com um procedimento similar ao utilizado na derivação do termo de difusão em condições tradicionais.

A seguinte relação pode ser escrita para a função estrutura Lagrangeana $D_{\mathrm{i}}(\tau)$ :

$$
D_{i}(\tau)=2 \sigma_{i}^{2}[1-R(\tau)]=C_{o} \varepsilon \tau \text {, }
$$

onde $R\left(\right.$ ) é a função de autocorrelação Euleriana, $\mathrm{C}_{0}$ é a constante de Kolmogorov e $\varepsilon$ é a taxa de dissipação da turbulência. Assumindo uma forma exponencial para $R(\tau)$ e expandindo em $\left(R(\tau)=1-\tau / \mathrm{T}_{\mathrm{L}}\right)$ Tennekes (1979) encontrou:

$$
\varepsilon=\frac{2 \sigma_{i}^{2}}{C_{o} T_{L}}
$$

Repetindo o mesmo procedimento, usando a expressão $R(\tau)$ (Eq. (1a) ) para o caso de vento fraco, obtêm-se:

$$
\varepsilon=\frac{2 \sigma_{i}^{2} p}{C_{o}}
$$

Recentemente, Anfossi et al. (2005b) propuseram um novo sistema de equações de Langevin acopladas baseado na chamada "solução simples de Thomson" (Thomson, 1987), para o caso geral de turbulência não-homogênea e condições estacionárias, mostrada abaixo:

$$
\begin{aligned}
& d u=\left\{-p(u-\bar{u})-q(v-\bar{v})+\frac{\partial \bar{u}}{\partial x} u+\frac{\partial \bar{u}}{\partial y} v+\sigma_{u} \frac{\partial \sigma_{u}}{\partial x}\right. \\
& \left.+\frac{(u-\bar{u})}{\sigma_{u}}\left[\frac{\partial \sigma_{u}}{\partial x} u+\frac{\partial \sigma_{u}}{\partial y} v\right]\right\} d t+\sqrt{2 p d t} \sigma_{u} \xi u \\
& d v=\left\{q(u-\bar{u})-p(v-\bar{v})+\frac{\partial \bar{v}}{\partial x} u+\frac{\partial \bar{v}}{\partial y} v+\sigma_{v} \frac{\partial \sigma_{v}}{\partial y}\right. \\
& \left.+\frac{(v-\bar{v})}{\sigma_{v}}\left[\frac{\partial \sigma_{v}}{\partial x} u+\frac{\partial \sigma_{v}}{\partial y} v\right]\right\} d t+\sqrt{2 p d t} \sigma_{v} \xi v
\end{aligned}
$$

onde $u$ e $v$ são as componentes horizontais das flutuações da velocidade do vento e $\bar{u}$ e $\bar{v}$ são as velocidades médias.

\subsection{O experimento de Inel}

O experimento de INEL foi realizado no Idaho National Engineering Laboratory (INEL) no sudeste de Idaho, EUA (Sagendorf e Dickson, 1974). O poluente SF6 foi liberado $1.5 \mathrm{~m}$ acima da superfície. Concentrações ao nível do solo foram medidas a $0.76 \mathrm{~m}$ por sessenta amostradores (colocados em 
Tabela 1 - Parâmetros meteorológicos estimados durante o experimento de INEL.

\begin{tabular}{|c|c|c|c|c|c|c|c|c|c|c|}
\hline $\operatorname{Exp}$ & $u *(\mathrm{~m} / \mathrm{s})$ & $L(\mathrm{~m})$ & $h(\mathrm{~m})$ & $U(2 \mathrm{~m})$ & $U(4 \mathrm{~m})$ & $U(8 \mathrm{~m})$ & $U(16 \mathrm{~m})$ & $U(32 \mathrm{~m})$ & $U(61 \mathrm{~m})$ & $\mathrm{Q}(\mathrm{g} / \mathrm{s})$ \\
\hline 4 & 0.047 & 2.40 & 13.40 & 0.7 & 1.2 & 999 & 1.5 & 0.9 & 2.1 & 0.032 \\
\hline 6 & 0.079 & 6.87 & 29.39 & 1.2 & 1.3 & 1.2 & 1.6 & 1.7 & 1.8 & 0.031 \\
\hline 7 & 0.040 & 1.77 & 10.64 & 0.6 & 0.9 & 1.2 & 0.5 & 0.9 & 2.4 & 0.033 \\
\hline 8 & 0.033 & 1.22 & 8.09 & 0.5 & 0.8 & 0.6 & 1.2 & 1.6 & 2.7 & 0.033 \\
\hline 9 & 0.033 & 1.22 & 8.09 & 0.5 & 0.8 & 0.9 & 1.6 & 2.2 & 2.7 & 0.032 \\
\hline 10 & 0.073 & 5.93 & 26.40 & 1.1 & 1.7 & 2.1 & 3.2 & 4.7 & 3.1 & 0.032 \\
\hline 11 & 0.093 & 9.60 & 37.91 & 1.4 & 1.9 & 2.3 & 2.9 & 999 & 3.6 & 0.031 \\
\hline 12 & 0.047 & 2.40 & 13.40 & 0.7 & 1.1 & 1.1 & 1.6 & 1.6 & 1.9 & 0.032 \\
\hline 13 & 0.067 & 4.90 & 22.88 & 1.0 & 1.6 & 2.0 & 3.0 & 4.0 & 6.0 & 0.033 \\
\hline 14 & 0.067 & 4.90 & 22.88 & 1.0 & 1.5 & 2.0 & 3.5 & 5.1 & 7.1 & 0.032 \\
\hline
\end{tabular}

intervalos de $6^{\circ}$ ), localizados ao longo de três arcos concêntricos $\left(360^{\circ}\right)$ com raios de 100,200 e $400 \mathrm{~m}$ em relação ao ponto de emissão (totalizando 180 amostradores). No experimento de INEL informações meteorológicas como velocidade, direção do vento e desvio padrão da direção do vento foram registradas em uma torre meteorológica em seis níveis $(2,4,8,16,32$ e $61 \mathrm{~m}$ ) com anemômetros tradicionais colocados sobre o arco de $200 \mathrm{~m}$. Na Tabela 1 são apresentados os dados meteorológicos utilizados para a validação do modelo proposto.

\subsubsection{Implementação no modelo}

O experimento de INEL ocorreu em um terreno plano, com a distância de $400 \mathrm{~m}$ sendo a maior separação entre o ponto de abandono e os amostradores. Além do mais, os dados de velocidade do vento foram observados em um único ponto, de modo que as variações horizontais deste observável não foram registradas. Considerando-se estas características é possível assumir homogeneidade horizontal e escrever a Equação 6 da seguinte forma (Anfossi et al., 2006):

$$
\begin{gathered}
d u=\{-p(u-\bar{u})-q(v-\bar{v})\} d t+\sqrt{2 p d t} \sigma_{u} \xi \\
d u=\{-p(u-\bar{u})-q(v-\bar{v})\} \\
d v=\{q(u-\bar{u})-p(v-\bar{v})\} d t+\sqrt{2 p d t} \sigma_{v} \xi v
\end{gathered}
$$

O modelo estocástico Lagrangeano LAMBDA tradicional (Brusasca et al., 1992; Ferrero e Anfossi, 1998 a,b) aplica-se a uma turbulência bem desenvolvida (vento forte) ocorrendo em uma superfície plana. Diferentemente, as Equações 7 podem ser empregadas em situações na qual o vento é fraco e existe a presença do fenômeno de meandro. Desta forma, o modelo matemático de dispersão representado pelas Equações 7 será chamado de LAMBDA LW ou LAMBDA low wind.

No experimento de INEL duas simulações foram consideradas usando o modelo LAMBDA: na primeira foram empregadas as equações de Langevin descritas pelas Equações 7, e que descrevem as componentes horizontais em condições de vento fraco; na segunda foi utilizado o modelo LAMBDA tradicional, que não leva em conta o fenômeno de meandro.

Para as duas simulações, a componente vertical da velocidade turbulenta $\omega$ foi calculada como usual, ou seja, resolvendo-se a seguinte equação de Langevin (Thomson, 1987)

$$
d w=a_{i}(z, w)+b_{o}(z) d W_{j}
$$

onde $d W_{j}$ é o processo Gaussiano incremental de Wiener (com média zero e variância $d t), b_{o}(z)=\sqrt{2 \sigma_{w}^{2} / T_{w}}\left(\sigma_{\mathrm{w}}^{2}\right.$ é a variância da flutuação da velocidade na vertical e $\mathrm{T}_{\mathrm{Lw}}$ é a escala de tempo Lagrangeana vertical) e $a_{i}(z, w)$ é calculado a partir da equação de Fokker-Planck associada a Equação 8, usando-se uma FDP Gram-Chalier truncada na terceira ordem (Ferrero e Anfossi, 1998 b).

A posição de cada partícula, em cada passo no tempo, é obtida pela integração numérica das Equações 7 e 8 a partir da seguinte equação: 


$$
d x_{i}=u_{i} d t
$$

onde $i=1,2,3, x_{i}$ é o vetor posição de cada partícula, $u_{i}$ é a combinação dos vetores velocidade do vento médio e velocidade Lagrangeana. Para modelar a difusão de escalares passivos na CLP, a equação de Langevin é resolvida seguindo as regras do cálculo proposto por Ito (Gardiner, 1985), que foi desenvolvido para se obter soluções das equações diferenciais estocásticas.

Em modelos de difusão atmosférica, uma parametrização adequada dos diferentes efeitos dispersivos é essencial. A confiabilidade de cada modelo depende fortemente do modo como os diferentes parâmetros associados aos fenômenos de transporte são calculados e relacionados ao desenvolvimento físico da CLP (Mangia et al., 2002). As quantidades $p$ e $q$ são os parâmetros físicos principais na descrição dos efeitos de meandro do vento nas Equações 7. Portanto, para se calcular $p$ e $q$ (dados pela Equação 1b) é necessário conhecer os parâmetros $m$ e $T$. Os valores para os parâmetros $m$ e $T$ são fornecidos pelas seguintes relações (Carvalho e Vilhena, 2005):

$$
\begin{aligned}
& m=\frac{8.5}{(1+U)^{2}}, \\
& T=\frac{m T_{*}}{2 \pi\left(m^{2}+1\right)}, \\
& T_{*}=200 m+500
\end{aligned}
$$

onde $U$ é a velocidade média do vento calculada a partir da média dos dados de velocidade do vento observados em diferentes alturas e $T_{+}$é o período de meandro. $\mathrm{O}$ comprimento de Monin-Obukhov $L$ (definindo uma altura na qual existe uma equivalência entre os termos de produção térmica e mecânica da energia cinética turbulenta) e a velocidade de fricção não foram medidos diretamente no experimento de INEL, mas podem ser aproximadamente estimados por diferentes formulações. Segundo Venkatram (1988) e Zannetti (1990), para uma camada limite estável, na qual a turbulência é gerada por um forçante mecânico, a escala característica $L$ pode ser estimada a partir da seguinte formulação:

$$
L=A u_{*}^{2}
$$

onde $A=1.1 \times 10^{3} \mathrm{~s}^{2} \cdot m^{-1}$. Por outro lado, a velocidade de fricção é obtida pela seguinte expressão:

$$
\mathrm{u}_{*}=\frac{\mathrm{ku}\left(\mathrm{z}_{\mathrm{r}}\right)}{\ln \left(\mathrm{z}_{\mathrm{r}} / \mathrm{z}_{\mathrm{o}}\right)} \text {, }
$$

onde $z_{r}=2 m$ (altura de referência) e $k$ é a constante de von Karman ( 0.4). Finalmente, as estimativas da altura $h$, para uma camada limite planetária estável, podem ser obtidas da seguinte relação sugerida por Zilitinkevich (1972),

$$
\mathrm{h}=0.4 \sqrt{\frac{\mathrm{u}_{*} \mathrm{~L}}{\mathrm{f}_{\mathrm{c}}}}
$$

onde $f_{\mathrm{c}}=0.0001 \mathrm{~s}^{-1}$ é o parâmetro de Coriolis. Cabe ressaltar que as formulações (13), (14) e (15) são válidas para uma turbulência gerada pelo efeito do cisalhamento do vento. Isto significa, que a turbulência mecânica existe na presença de um fluxo turbulento de calor sensível negativo. Os valores de $\sigma_{\mathrm{i}}{ }^{2} \mathrm{e}$ $\tau_{L i}$ foram calculados de acordo com a parametrização derivada por Degrazia et al. (2000). Seguindo a formulação proposta por Degrazia et al. (2000), os valores de $L$ e $u *$ são utilizados para se calcular os valores das variâncias das velocidades e das escalas de tempo Lagrangeanas.

No modelo LAMBDA LW e LAMBDA tradicional, o domínio horizontal foi determinado pela distância dos amostradores. Diferentemente, o domínio vertical foi considerado igual à altura $h$ da camada limite estável. A fonte foi localizada no centro do domínio. Um passo de tempo, $\Delta t=$ $0,5 \mathrm{~s}$, foi mantido constante durante as simulações. A magnitude deste passo de tempo é da ordem das escalas de tempo do subintervalo inercial de um espectro de energia turbulenta do tipo de Kolmogorov. Além do mais, este passo de tempo obedece à desigualdade $\Delta t<<\tau_{\mathrm{L}}$. Esta condição garante que as acelerações não sejam correlacionadas durante as escalas de tempo características do subintervalo inercial (Rodean, 1996). A partir da fonte, cem partículas foram liberadas em cada passo de tempo. Um total de 1500 passos de tempo foi empregado em cada simulação, gerando um número de partículas fictícias da ordem de 105. Esta magnitude permite a geração de médias confiáveis de concentração de contaminantes. $\mathrm{O}$ volume que representa a fonte pontual foi de $0.1 \mathrm{~m} \times 0.1 \mathrm{~m} \times 0.1 \mathrm{~m}$.

As concentrações normalizadas, $\chi_{m}\left(\mathrm{~m}^{-2}\right)$, foram calculadas como em Sagendorf e Dickson (1974), isto é

$$
\chi_{m}=\mathrm{C}_{m} \frac{\mathrm{U}_{4}}{\mathrm{Q}},
$$

onde $\mathrm{C}_{m}$ é a concentração expressa em $\mathrm{gm}^{-3}, \mathrm{U}_{4}$ é a velocidade do vento médio em $4 \mathrm{~m}$ e $Q$ é a taxa de emissão $\left(\mathrm{gs}^{-1}\right)$. Conseqüentemente, as concentrações simuladas para o experimento de INEL foram expressas em $\left(\mathrm{m}^{-2}\right)$. No caso dos experimentos do projeto INEL as células de concentração ao nível do solo possuem uma dimensão vertical de $\Delta z=3 m$. As dimensões horizontais foram computadas a partir da seguinte forma

$$
\Delta x=\Delta y=\frac{2 \pi r}{N_{\text {ang }}}
$$


onde $\mathrm{r}$ é o raio do arco e $\mathrm{N}_{\mathrm{ang}}=60$ é o número de amostradores por arco. Este modo de calcular o tamanho da célula permite cobrir todos os círculos com seus três raios sem uma significante superposição. Com estas considerações, os valores de $d x=d y$ foram respectivamente $10.47,20.93$ e $41.87 \mathrm{~m}$ para os três arcos.

\section{RESULTADOS E DISCUSSÃO}

O desempenho dos modelos LAMBDA LW e LAMBDA tradicional é apresentado nas Figuras 1-4 e na Tabela 2. As Figuras 1-4 demonstram os resultados típicos obtidos com o modelo LAMBDA LW e o modelo LAMBDA tradicional. A primeira (Figura 1) refere-se ao experimento 8, no qual a dispersão da pluma ocorre em todas as direções, ou seja, o traçante é coletado em todos os ângulos. A comparação entre os resultados das duas simulações torna evidente que o modelo LAMBDA LW é capaz de dispersar a pluma em todos os $360^{\circ}$, enquanto a simulação com o modelo LAMBDA produz uma pluma muito estreita. Além disso, enquanto a concentração máxima é aproximadamente reproduzida pelo novo modelo (LAMBDA LW), o modelo LAMBDA tradicional reproduz valores que superestimam este valor pico para a concentração. Considerando a Figura 2 pode-se novamente observar, que o novo modelo reproduz melhor a concentração máxima observada. Ainda, para este arco de 100m o modelo LAMBDA tradicional superestima o valor máximo da concentração e apresenta uma dispersão de contaminantes provocada pela ação de turbilhões pequenos associados à turbulência presente na camada limite estável. Adicionalmente, observa-se o mesmo comportamento quando comparamos os resultados obtidos a partir das Figuras 3 e 4, as quais se referem aos experimentos 11 e 12 , respectivamente.

Sabe-se que a dispersão atmosférica ocorrendo em condições de velocidade do vento muito baixa é principalmente governada pelo fenômeno de meandro do vento horizontal. Em tais situações, diferentemente do processo difusivo gerado por uma turbulência bem desenvolvida, a ação das oscilações de baixa freqüência do vento horizontal dispersa os contaminantes sobre os diferentes setores angulares. Desta forma, este comportamento oscilatório é o mecanismo físico que espalha os contaminantes nos diferentes ângulos e provoca uma redução na concentração máxima. O modelo LAMBDA LW, contendo os parâmetros descrevendo o efeito do meandro, consegue reproduzir o efeito descrito acima. Diferentemente, o modelo LAMBDA tradicional não é capaz de capturar os efeitos do meandro e descreve apenas um processo de difusão turbulenta que não contem a ação das oscilações de baixa freqüência do vento horizontal. Como uma conseqüência, os parâmetros físicos que compõem este último modelo, ou seja, $\sigma_{i}$ e $\tau_{L i} \mathrm{e}$ que foram obtidos para uma camada limite estável, promovem uma difusão turbulenta em pequena escala. Este último efeito é o mecanismo físico responsável pela difusão reduzida, que não permite uma maior abertura da pluma de contaminantes.

A Tabela 2 mostra os resultados da análise estatística de desempenho do modelo feita com os picos de concentração $(n=30)$, além de apresentar a comparação entre os índices estatísticos obtidos pelo modelo simulado e outros cinco trabalhos disponíveis na literatura. Os índices estatísticos foram calculados como sugeridos por Hanna (1989):

$$
\begin{aligned}
& \text { NMSE }=\overline{\left(\mathrm{C}_{\mathrm{o}}-\mathrm{C}_{\mathrm{p}}\right)^{2}} / \overline{\mathrm{C}_{\mathrm{o}} \mathrm{C}_{\mathrm{p}}} \text { (Erro Quadrático Médio Normalizado) } \\
& \mathrm{FB}=\left(\overline{\mathrm{C}_{\mathrm{o}}}-\overline{\mathrm{C}_{\mathrm{p}}}\right) /\left(0.5\left(\overline{\mathrm{C}_{\mathrm{o}}}+\overline{\mathrm{C}_{\mathrm{p}}}\right)\right) \\
& \text { (Desvio Fracional) } \\
& \mathrm{FS}=2\left(\sigma_{0}-\sigma_{\mathrm{p}}\right) /\left(\sigma_{0}+\sigma_{\mathrm{p}}\right) \\
& \text { (Desvio Padrão Fracional) } \\
& \mathrm{COR}=\left(\overline{\left.\mathrm{C}_{\mathrm{o}}-\overline{\mathrm{C}_{\mathrm{o}}}\right)\left(\mathrm{C}_{\mathrm{p}}-\overline{\mathrm{C}_{\mathrm{p}}}\right)} / \sigma_{0}+\sigma_{\mathrm{p}} \quad\right. \text { (Coeficiente de Correlação) }
\end{aligned}
$$$$
\mathrm{FA} 2=0.5,<\mathrm{C}_{\mathrm{o}} / \mathrm{C}_{\mathrm{p}}<2
$$

(Fator de 2)

onde $C$ é a quantidade analisada (concentração) e os subscritos "o" e "p" representam os valores observados e previstos, respectivamente. $F B$ indica a tendência do modelo subestimar ou superestimar as concentrações observadas, NMSE fornece a informação dos desvios entre a concentração prevista e observada, $F S$ indica o quanto o modelo consegue simular a dispersão dos dados observados, $C O R$ indica o quanto $C_{0}$ e $C_{P}$ estão relacionados e $F A 2$ fornece a fração dos dados para os quais $0.5 \leq C_{0} / C p \leq 2$.

Os resultados da Tabela 2 e das Figuras 1-4 mostram boa concordância entre os valores observados e simulados pelo modelo LAMBDA LW. De acordo com a Tabela 2 , nota-se que os valores dos índices estatísticos NMSE, FB e FS estão próximos de zero e COR e FA2 estão próximos de 1. Mais especificamente, pode-se perceber que o modelo superestimou levemente os valores de concentração $(F B=-0.04)$, apresenta uma alta correlação entre os dados observados e simulados $(C O R=0.89)$ e mostra que a dispersão dos valores de concentração simulados é somente um pouco maior que a dispersão dos valores de concentração observados ( $\mathrm{FS}=-$ 0.053). Considerando, ainda, a Tabela 2 verifica-se que os resultados obtidos a partir do modelo LAMBDA tradicional não são satisfatórios, demonstrando, então, que os modelos de dispersão tradicionais não levam em conta o efeito de meandro do vento.

Além disso, comparando os índices estatísticos obtidos pelo modelo LAMBDA LW com os obtidos por outros modelos percebe-se uma leve melhora em alguns casos. Porém, o modelo de Oettl et al. (2001) e o modelo proposto neste artigo tem a vantagem de poderem ser aplicados a todos os tipos de fontes e 
condições de campos de vento não-homogêneos, em contraste aos modelos de Sagendorf e Dickson (1974), Sharan e Yadav (1998), os quais utilizaram modelos de pluma Gaussiana.

A fim de compararmos os nossos resultados obtidos com os resultados obtidos por Anfossi et al. (2006), os quais simularam o experimento de INEL com o mesmo conjunto de equações de Langevin para as componentes longitudinais utilizadas neste artigo, e para ilustrar as diferenças entre as duas opções de simulações propostas aqui, os seguintes

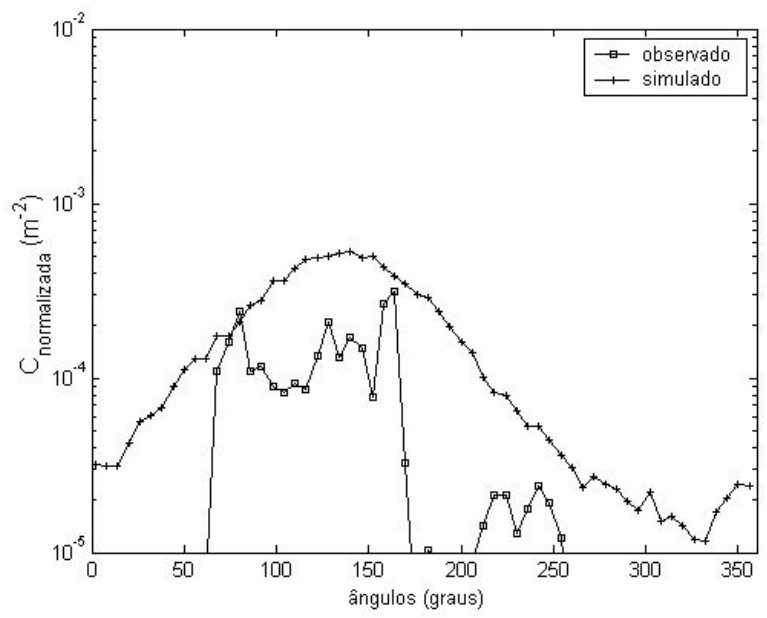

(a) índices estatísticos foram calculados para cada arco dos dez experimentos: concmax, top5, $C y$ e $S y$. Concmax $\left(\mathrm{m}^{-2}\right)$ é a concentração máxima ao nível do solo, top5 $\left(\mathrm{m}^{-2}\right)$ referese à média dos cinco valores mais altos de concentração experimental e simulada ao nível do solo, $C y=\Delta y \sum_{i=1}^{N} \chi_{i}\left(\mathrm{~m}^{-1}\right)$ é a concentração ao nível do solo integrada perpendicular a direção do vento e

$$
S Y=\sqrt{\sum_{i=1}^{N}\left(\vartheta_{i}-\bar{\vartheta}\right)^{2} / \sum_{i=1}^{N} \chi_{i}}
$$

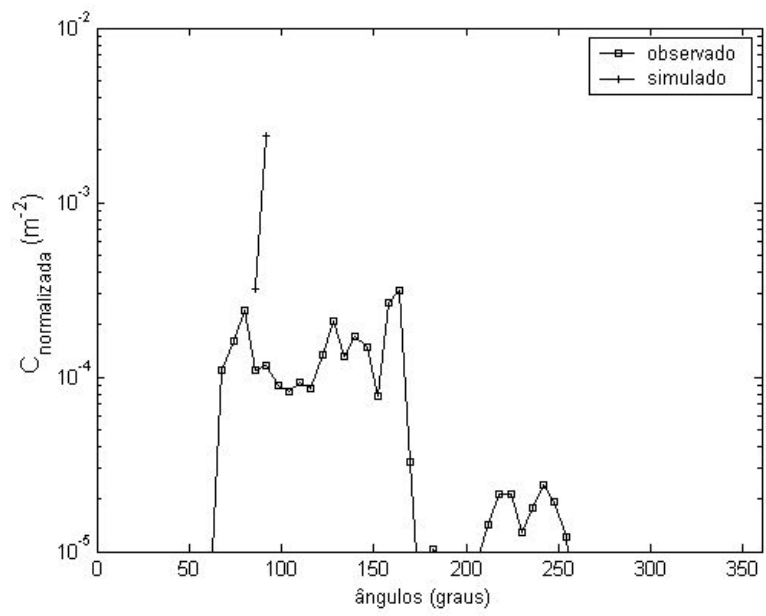

(b)

Figura 1 - Concentração ao nível do solo normalizada $\left(\mathrm{m}^{-2}\right)$ em função dos ângulos para o experimento 8 a $200 \mathrm{~m}$. As figuras plotadas referem-se ao (a) modelo LAMBDA LW e (b) modelo LAMBDA tradicional. A linha ( $\square$ ) indica concentração observada e a linha $(\times)$ indica concentração simulada.

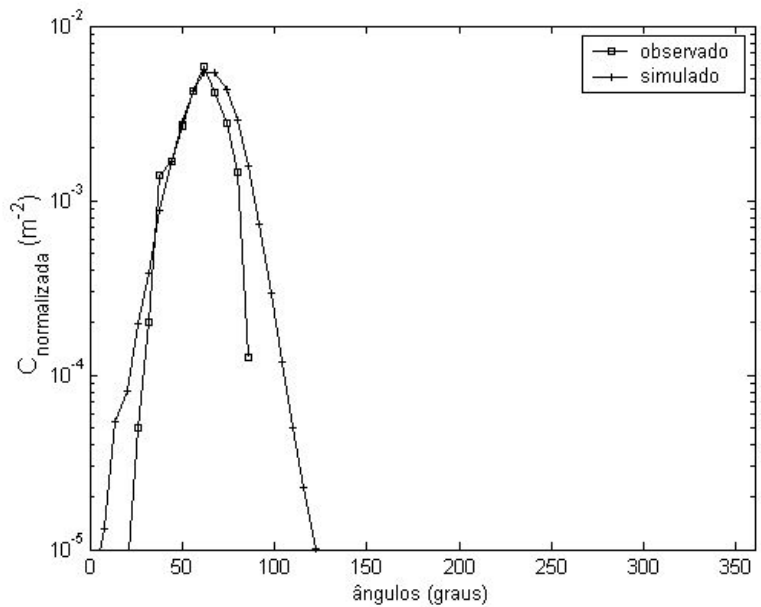

(a)

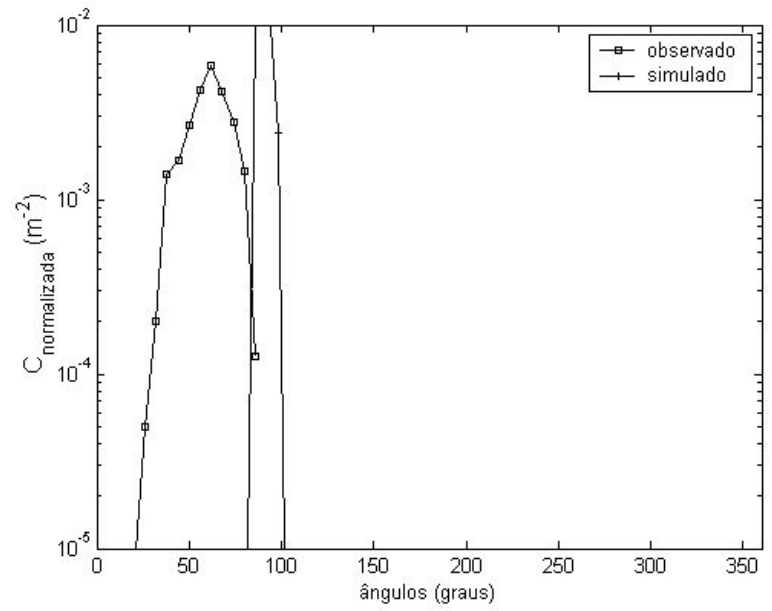

(b)

Figura 2 - Concentração ao nível do solo normalizada $\left(\mathrm{m}^{-2}\right)$ em função dos ângulos para o experimento 4 a $100 \mathrm{~m}$. As figuras plotadas referem-se ao (a) modelo LAMBDA LW e (b) modelo LAMBDA tradicional. A linha ( $\square$ ) indica concentração observada e a linha $(\times)$ indica concentração simulada. 


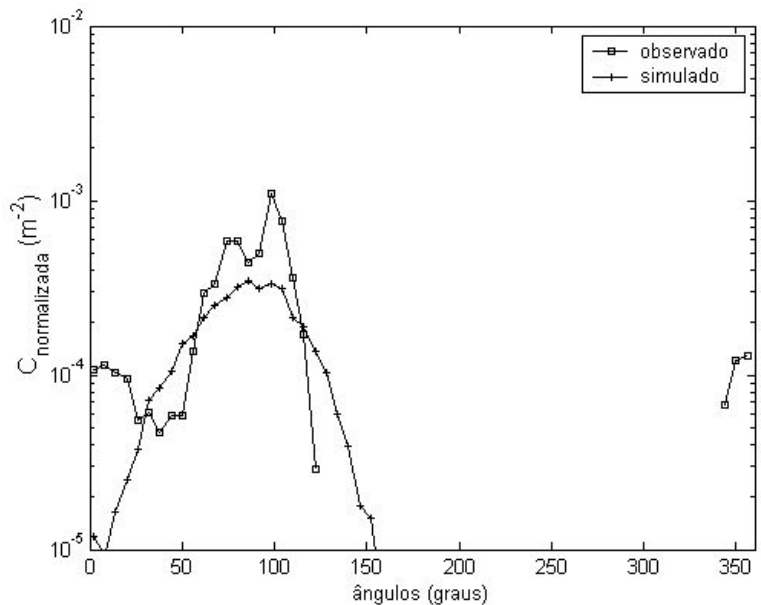

(a)

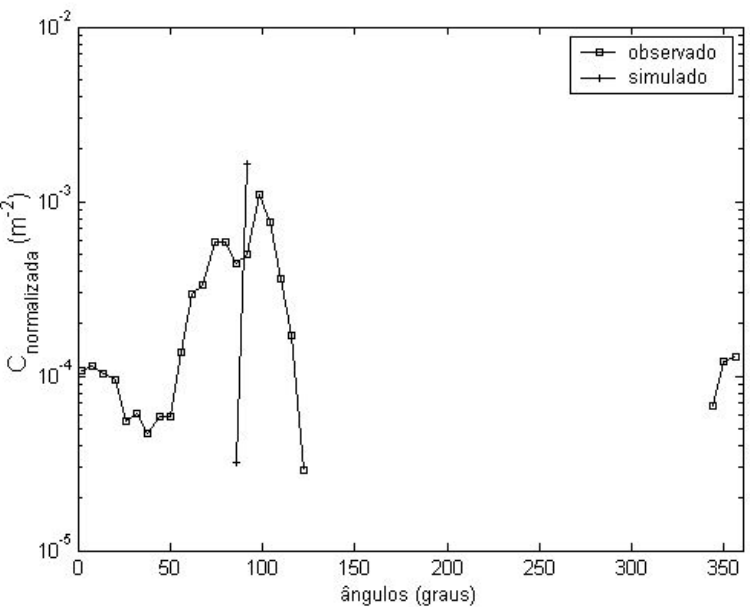

(b)

Figura 3 - Concentração ao nível do solo normalizada $\left(\mathrm{m}^{-2}\right)$ em função dos ângulos para o experimento 11 a $400 \mathrm{~m}$. As figuras plotadas referemse ao (a) modelo LAMBDA LW e (b) modelo LAMBDA tradicional. A linha ( $\square$ ) indica concentração observada e a linha $(\times)$ indica concentração simulada.

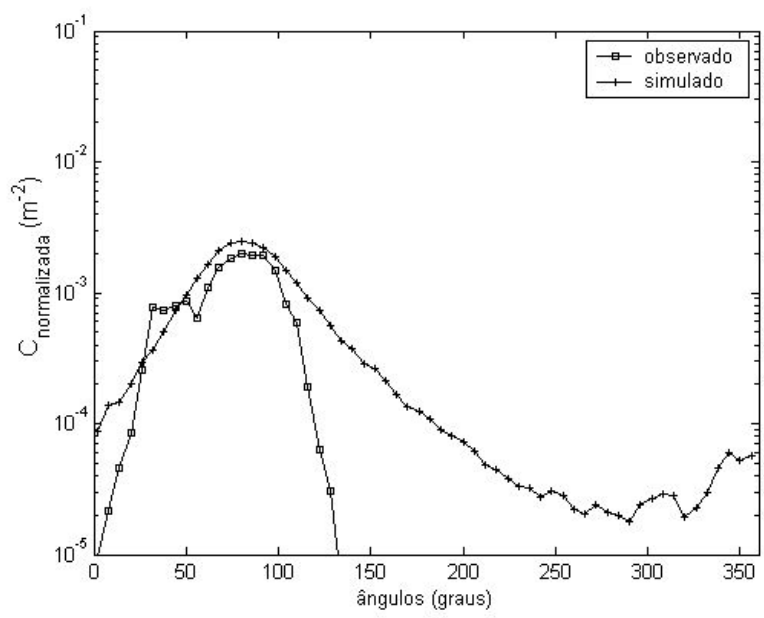

(a)

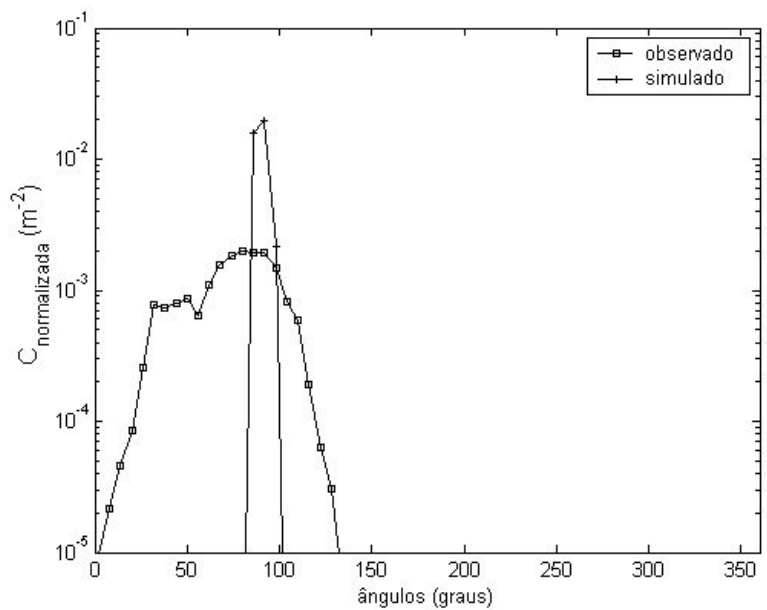

(b)

Figura 4 - Concentração ao nível do solo normalizada $\left(\mathrm{m}^{-2}\right)$ em função dos ângulos para o experimento 12 a $100 \mathrm{~m}$. As figuras plotadas referemse ao (a) modelo LAMBDA LW e (b) modelo LAMBDA tradicional. A linha ( $\square$ ) indica concentração observada e a linha $(\times)$ indica concentração simulada.

Tabela 2 - Índices estatísticos de desempenho do modelo LAMBDA para o experimento de INEL obtidos pelos modelos simulados, por Sagendorf e Dickson (1974), por Sharan e Yadav (1998) e por Oettl et al. (2001).

\begin{tabular}{|l|c|c|c|c|c|}
\hline & NMSE & COR & FA2 & FB & FS \\
\hline LAMBDA LW & 0.17 & 0.89 & 0.77 & -0.04 & -0.053 \\
\hline LAMBDA & 7.38 & 0.41 & 0.067 & -1.40 & -1.44 \\
\hline Sagendorfe Dickson (1974) & 0.60 & 0.42 & 0.80 & 0.06 & - \\
\hline Sharan e Yadav (1998) & 0.53 & 0.55 & 0.60 & -0.02 & - \\
\hline Oettl et al. (2001) & 0.21 & 0.86 & 0.87 & -0.13 & - \\
\hline
\end{tabular}


onde $\vartheta_{i}$ são os ângulos dos amostradores no local onde foram realizadas as medidas das concentrações e $\bar{\vartheta} \mathrm{o}$ valor médio destes.

As Figuras 5-8 exibem uma comparação dos índices estatísticos das simulações do experimento de INEL realizadas pelo o modelo LAMBDA LW e LAMBDA tradicional. A Figura 5 mostra a comparação entre os valores simulados e observados de concmax. A diferença no desempenho dos modelos é bastante visível. Apesar de existir alguma dispersão, nota-se que os valores de concentração máxima simulados com o modelo LAMBDA LW alinham-se aproximadamente com a linha central de perfeita concordância. No caso do modelo LAMBDA tradicional a maior parte dos dados são superestimados. Os mesmos comentários feitos sobre as Figuras 1 a 4 são apropriados aqui também, ou seja, simulações do modelo LAMBDA tradicional não levam em conta o fenômeno de meandro do vento e os seus efeitos sobre a dispersão em condições de vento fraco, o que conduz a resultados não representativos da pluma de dispersão.

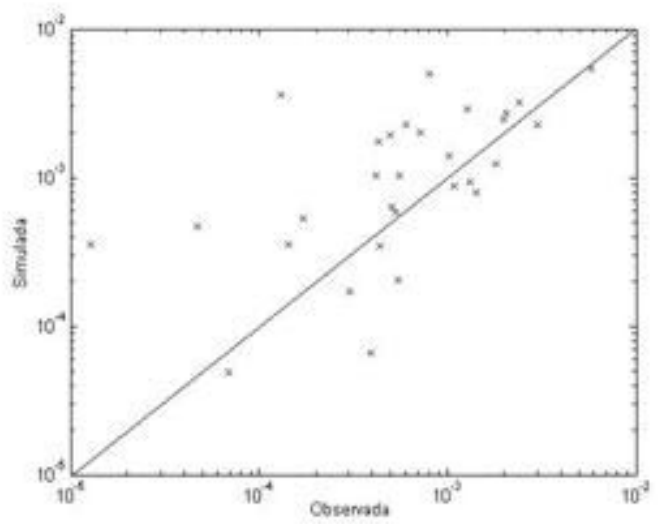

(a)

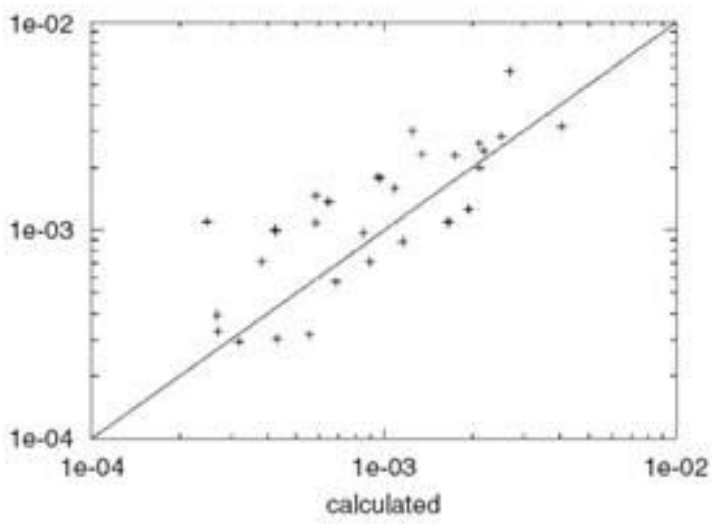

(c)
A Figura 6 apresenta a comparação entre os valores de concentração simulados e observados para os casos classificados como top5. As mesmas considerações feitas para a figura anterior também se aplicam neste caso. Para o caso simulado com o modelo LAMBDA tradicional, a distribuição da concentração ao nível do solo superestima os valores de concentração observados.

A Figura 7 mostra a comparação entre os valores simulados (modelo LAMBDA LW e modelo LAMBDA tradicional) e observados de $C y$. Neste caso, não existe diferenças significativas entre as duas simulações, visto que ambos os valores de concentração alinham-se aproximadamente a linha central, a qual representa a concordância entre os dados de concentração simulados e observados. Esta boa comparação para ambos os modelos resulta da definição da concentração integrada lateralmente $C y$, ou seja, a integração no plano horizontal que fornece $C y$ mascara o efeito das oscilações de baixa freqüência associadas ao meandro e que são responsáveis pela dispersão

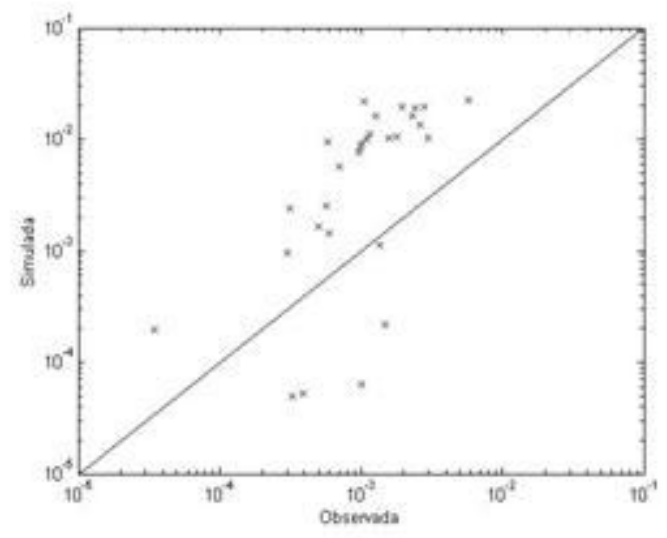

(b)

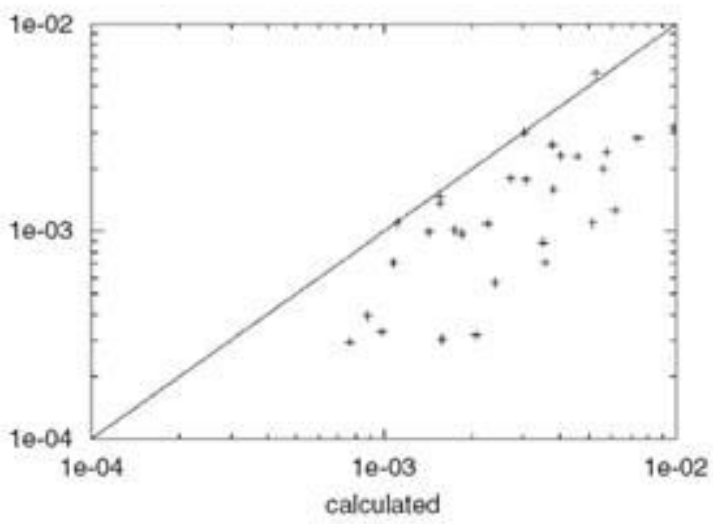

(d)

Figura 5 - Concentração máxima calculada com o (a) modelo LAMBDA LW e (b) modelo LAMBDA tradicional. Além disso, são apresentados os resultados obtidos por Anfossi et al. (2006) para (c) modelo LAMBDA low wind e (d) modelo LAMBDA tradicional. O eixo x mostra os valores observados e o eixo y os valores simulados. 
angular lateral da pluma de contaminantes. Desta maneira, o efeito dispersivo que determinou a concentração $C y$ é provocado pela turbulência bem desenvolvida (apresentando escala pequena em relação àquela provocada pelo meandro do vento).

A Figura 8 mostra a comparação entre os valores simulados e observados para $S y$. Neste caso, novamente, o efeito da simulação com o modelo LAMBDA LW torna-se claramente visível. O desvio padrão angular da concentração simulada pelo modelo LAMBDA tradicional é superestimada, enquanto o novo modelo simula aproximadamente bem estes dados.

É importante ressaltar que no conjunto formado pelas Figuras 5-8 estão contidas simulações que foram realizadas por Anfossi et al. (2006). Comparando-se o novo conjunto de índices estatísticos calculados percebe-se que o modelo LAMBDA tradicional não apresenta precisão nas simulações

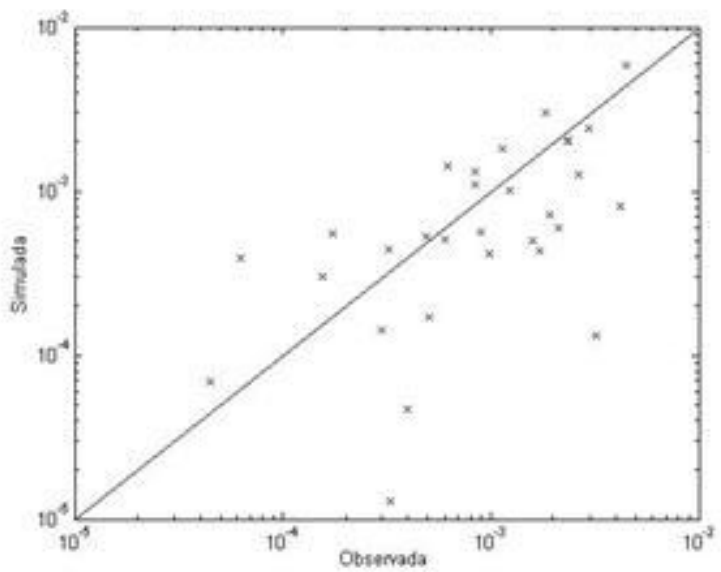

(a)

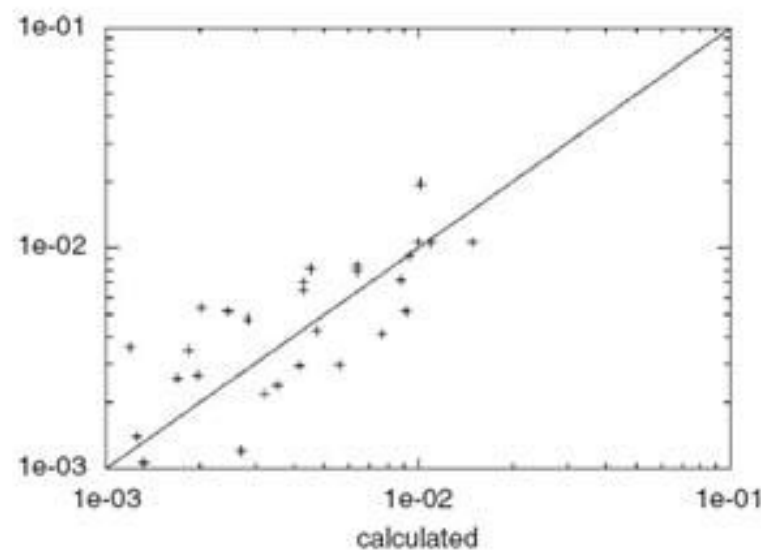

(c) envolvendo o fenômeno de meandro do vento. No entanto, o modelo LAMBDA LW apresenta boa concordância tanto com os valores de concentrações observadas, quanto com as concentrações reproduzidas nas simulações de Anfossi et al. (2006). A diferença entre o modelo LAMBDA LW, utilizado neste artigo, e o modelo de Anfossi et al. (2006) encontra-se na parametrização de $p$ e $q$, os quais estão contidos na função de autocorrelação Euleriana (Eqs. 1). Anfossi et al. (2006) calcularam os valores de $p$ e $q$ ajustando a equação para a função de autocorrelação Euleriana obtida a partir de dados experimentais, sendo que os diferentes pares de $p$ e $q$ foram estimados para cada experimento e para cada altura da torre $(2,4$, $8,16,32$ e $61 \mathrm{~m}$ ). Diferentemente, no presente estudo os valores das quantidades $p$ e $q$ foram calculados a partir dos parâmetros $m$ e $T$ propostos por Carvalho e Vilhena (2005).

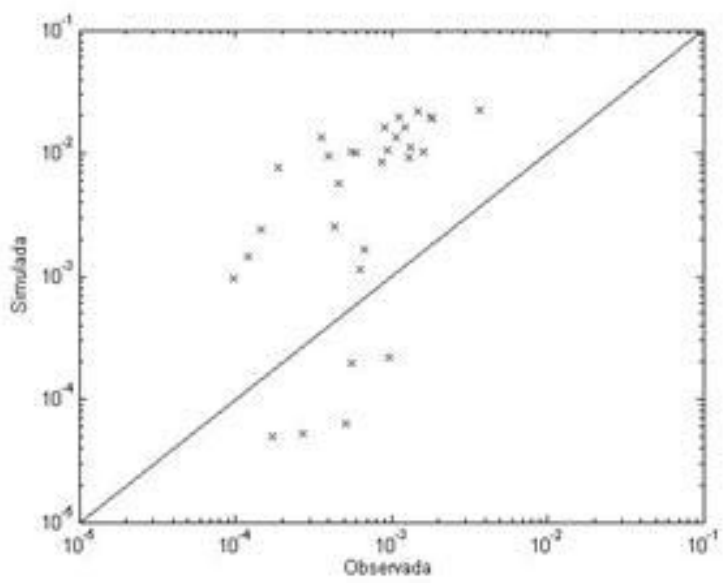

(b)

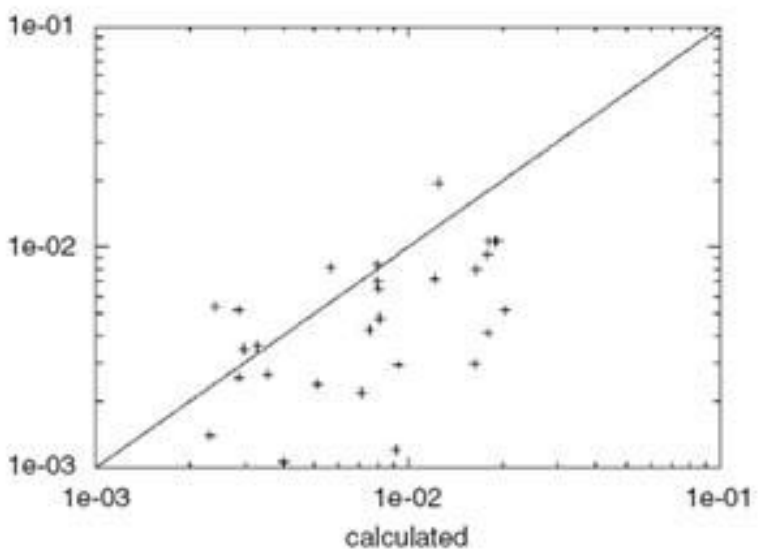

(d)

Figura 6 - Top5 calculada com o (a) modelo LAMBDA LW e (b) modelo LAMBDA tradicional. Além disso, são apresentados os resultados obtidos por Anfossi et al. (2006) para (c) modelo LAMBDA low wind e (d) modelo LAMBDA tradicional. O eixo x mostra os valores observados e o eixo y os valores simulados. 


\section{CONCLUSÕES}

Neste trabalho foi apresentado um estudo da dispersão de poluentes passivos, liberados de forma contínua, utilizando um modelo de partículas estocástico Lagrangeano com o objetivo de estimar a concentração de contaminantes em condições estáveis com velocidade do vento fraco. O modelo de partículas estocástico Lagrangeano utilizado foi o LAMBDA, o qual é baseado na forma tridimensional da equação de Langevin para a velocidade aleatória. A implementação do modelo LAMBDA permite levar em conta situações de escoamento os quais apresentam velocidade do vento fraco e variações temporais dos campos meteorológicos.

A nova versão do modelo LAMBDA leva em conta um sistema de equações de Langevin acopladas para as

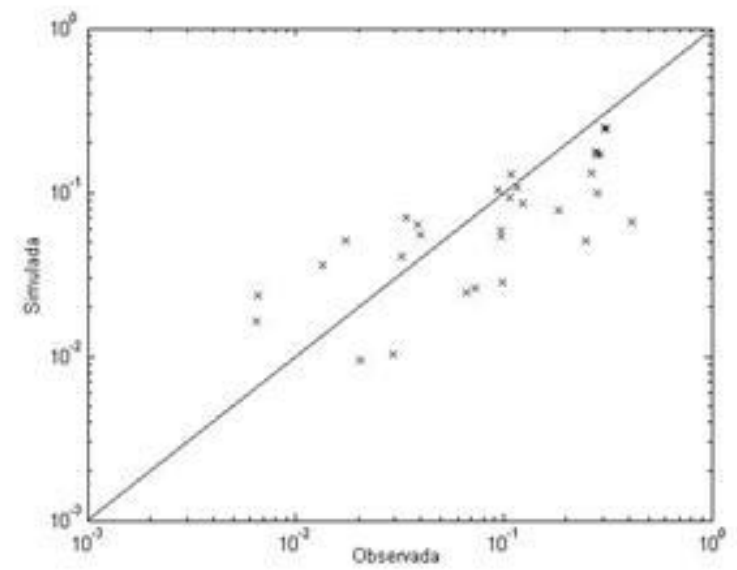

(a)

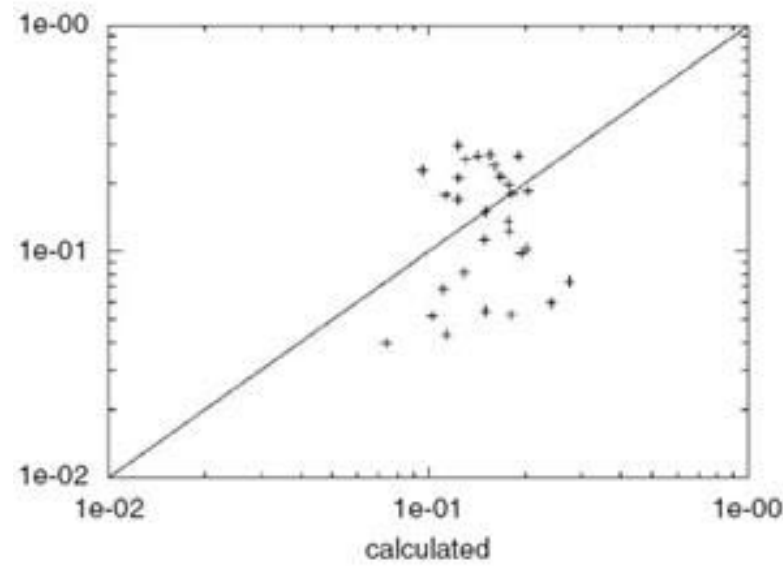

(c) componentes horizontais do vento que considera os efeitos de meandro do vento. Tais equações foram propostas por Anfossi et al. (2005b) e, recentemente, testadas por Anfossi et al. (2006) obtendo resultados satisfatórios. Além disso, foi sugerida uma nova parametrização para os parâmetros $p$ e $q$ para o cálculo das equações de Langevin como um valor constante e dependente apenas da velocidade média do vento para cada experimento. As simulações foram repetidas utilizando o modelo LAMBDA tradicional, com o objetivo de verificar a melhora na precisão, obtida com o novo sistema de equações para as componentes longitudinais. Estas somente diferem nas componentes horizontais da equação de Langevin, todas as outras especificações e dados de entrada seguem os mesmos.

Particularmente, os resultados obtidos com o novo sistema de equações concordam muito bem com os dados

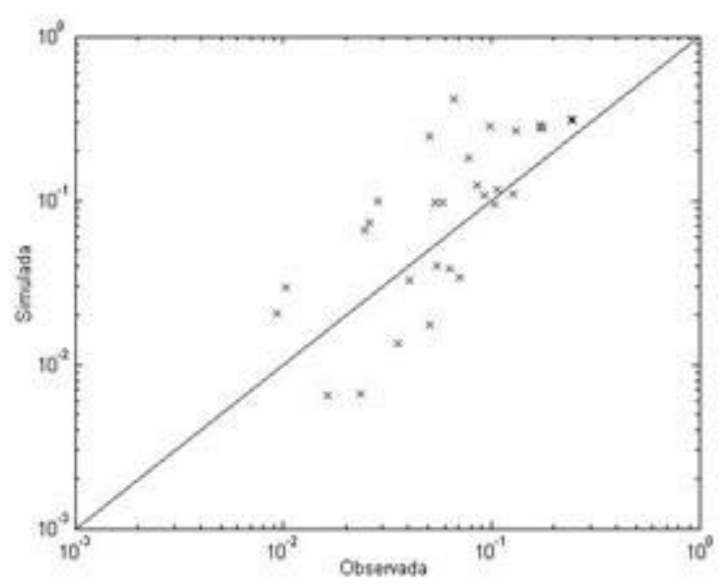

(b)

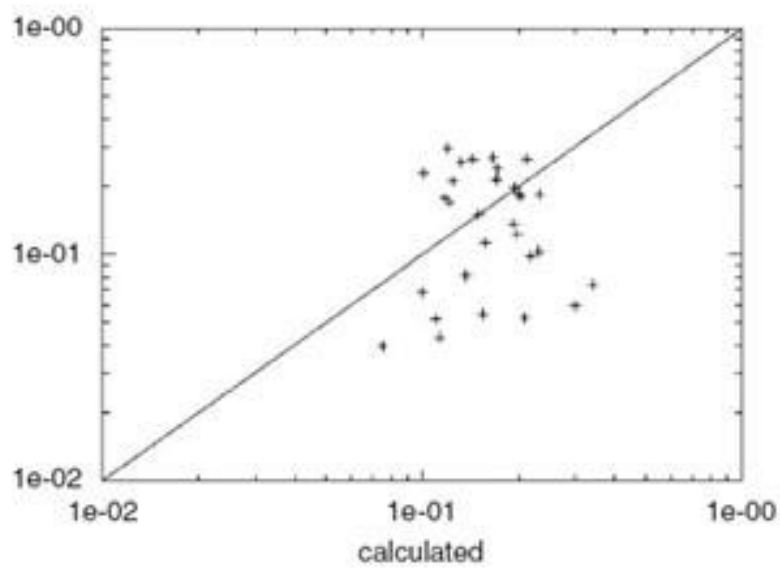

(d)

Figura 7 - Concentração integrada ( ) calculada com o (a) modelo LAMBDA LW e (b) modelo LAMBDA tradicional. Além disso, são apresentados os resultados obtidos por Anfossi et al. (2006) para (c) modelo LAMBDA low wind e (d) modelo LAMBDA tradicional.O eixo x mostra os valores observados e o eixo y os valores simulados. 
experimentais, indicando que o modelo representa o processo de dispersão corretamente em condições de velocidade do vento fraco. Diferentemente do processo difusivo gerado por uma turbulência bem desenvolvida, a ação das oscilações de baixa freqüência do vento horizontal dispersa os contaminantes sobre os diferentes setores angulares. Desta forma, este comportamento oscilatório é o mecanismo físico que espalha os contaminantes nos diferentes ângulos e provoca uma redução na concentração máxima. Assim, demonstrando que esse novo conjunto de equações para as componentes horizontais pode ser incorporado a um sistema de modelos para estimar a qualidade do ar. Por outro lado, considerando o modelo LAMBDA tradicional, este não é capaz de capturar os efeitos do meandro e descreve apenas um processo de difusão turbulenta que não contem a ação das oscilações de baixa freqüência do vento horizontal.

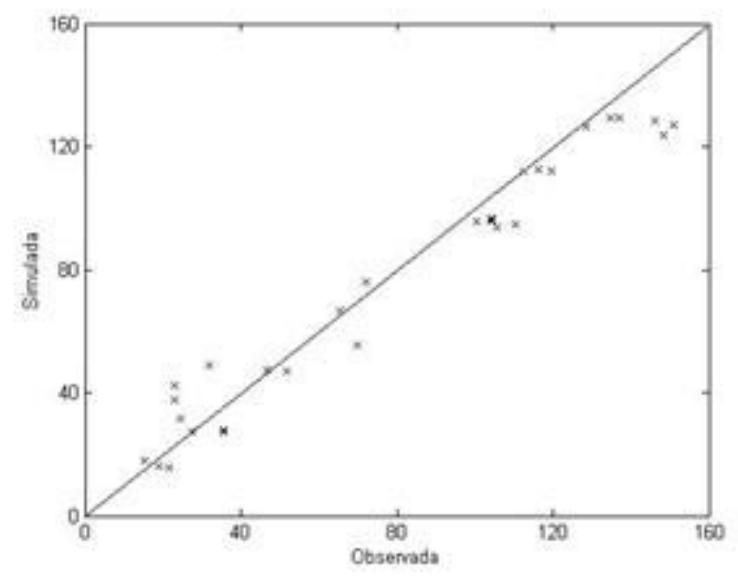

(a)

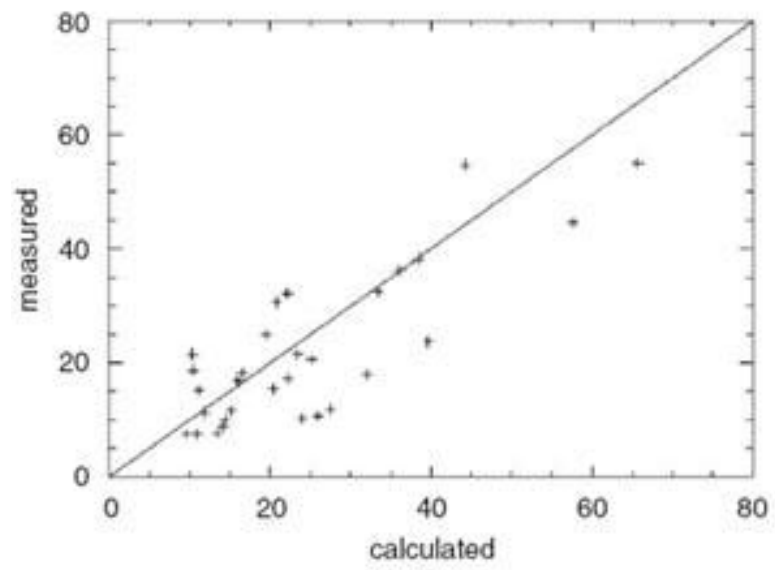

(c)
A principal conclusão deste trabalho foi verificar a qualidade da nova parametrização dos parâmetros $p$ e $q$ quando aplicados ao sistema de equações de Langevin proposto para as componentes horizontais, ou seja, demonstrar que valores de $p$ e $q$ constantes podem gerar resultados satisfatórios na simulação da dispersão de contaminantes. Anfossi et al. (2006) calcularam os valore de $p$ e $q$ fitando a equação para a função de autocorrelação Euleriana obtida a partir de dados experimentais, estimando diferentes pares de $p$ e $q$ para cada experimento e para cada altura da torre $(2,4,8,16,32$ e $61 \mathrm{~m})$. No entanto, este último, apesar de apresentar resultados satisfatórios, pode ser considerado muito mais trabalhoso, do ponto de vista das simulações.

Portanto, a vantagem do modelo LAMBDA LW é que este pode ser aplicado a todos os tipos de turbulência presentes

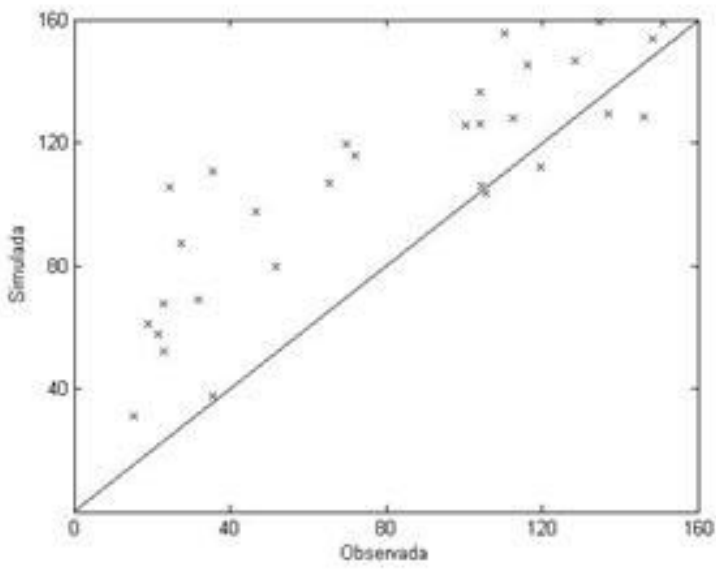

(b)

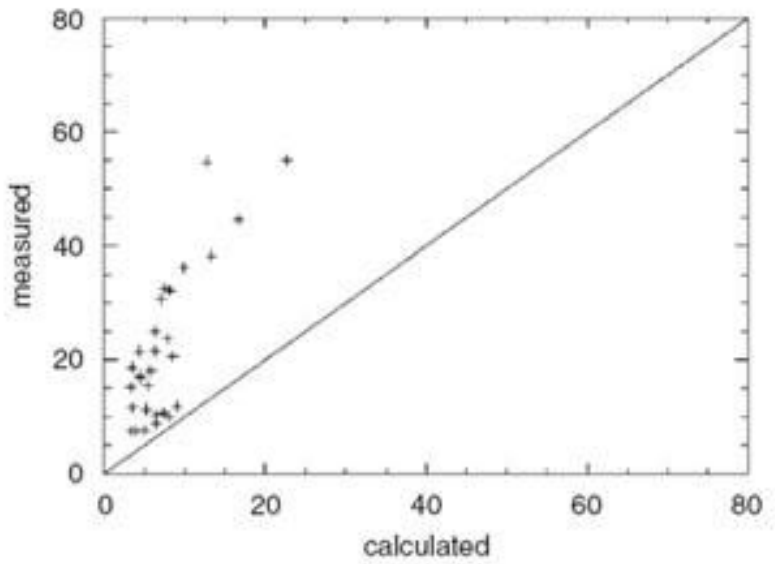

(d)

Figura 8 - Sy calculado com o (a) modelo LAMBDA LW e (b) modelo LAMBDA tradicional. Além disso, são apresentados os resultados obtidos por Anfossi et al. (2006) para (c) modelo LAMBDA low wind e (d) modelo LAMBDA tradicional. O eixo x mostra os valores observados e o eixo y os valores simulados. 
na CLP, ou seja, em casos de turbulência bem desenvolvida e em casos de turbulência associada ao efeito de meandro do vento (turbulência fraca). Isso é demonstrado quando consideramos $m=0$ (ou seja, quando o efeito de meandro é eliminado) na Equação 1b, a qual reproduz a expressão clássica para a função de autocorrelação Euleriana experimental, normalmente utilizada na modelagem da dispersão em condições de vento forte.

\section{AGRADECIMENTOS}

Os autores agradecem ao suporte financeiro fornecido pela CAPES (Coordenação de Aperfeiçoamento de Pessoal de Nível Superior) e ao CNPq (Conselho Nacional de Desenvolvimento Científico e Tecnológico).

\section{REFERÊNCIAS BIBLIOGRÁFICAS}

ANFOSSI, D.; ALESSANDRINI, S.; TRINI CASTELLI, S.; FERRERO, E.; OETTL, D.; DEGRAZIA, G. Tracer dispersion simulation in low wind speed conditions with a new 2D Langevin equation system. Atmospheric Environment, v.40, p.7234-7245, 2006.

ANFOSSI, D.; OETTL, D.; DEGRAZIA, G.; GOULART, A. An analysis of sonic anemometer observations in low wind speed conditions. Boundary-Layer Meteorology, v.114, p.179-203, 2005a.

ANFOSSI, D.; TINARELLI, G.; TRINI CASTELLI, S.; FERRERO, E.; OETTL, D.; DEGRAZIA, G.; MORTARINI, L. Well mixed condition verification in windy and low wind speed conditions. Proceedings of Harmo 10, Create, 17-20 October 2005 b.

BRUSASCA, G.; TINARELLI, G.; ANFOSSI, D. Particle model simulation of diffusion in low wind speed stable conditions. Atmospheric Environment, v.26A, p.707-723, 1992.

CARVALHO, J.C.; VILHENA, M.T. Pollutant dispersion simulation for low wind speed condition by the ILS method. Atmospheric Environment, v. 39, p. 6282-6288, 2005.

CIRILLO, M.C.; POLI, A.A. An intercomparison of semiempirical diffusion models under low wind speed, stable conditions. Atmospheric Environment, v.26A, p.765774, 1992.

DEGRAZIA, G. A.; ANFOSSI, D.; CARVAlHO, J. C.; MANGIA, C.; TIRABASSI, T.; CAMPOS VELHO, H. F. Turbulence parameterization for PBL dispersion models in all stability conditions. Atmospheric Environment, v. 34, p. 3575-3583, 2000.

FERRERO, E.; ANFOSSI, D. Sensitivity analysis of Lagrangian stochastic models for CBL with different
FDPs and turbulence parameterizations. In: Air Pollution Modelling and its Applications XII, GRYNING, S.E.; N. CHAUMERLIAC (Eds.), Plenum Press, New York, p. 673-368, 1998a.

FERRERO, E.; ANFOSSI, D. Comparison of FDPs, closure schemes and turbulence parameterizations in Lagrangian stochastic models. International Journal of Environment and Pollution, v.9, p. 384-410, 1998 b.

FRENKIEL, F.N. Turbulent diffusion: mean concentration distribution in a flow field of homogeneous turbulence. Advances in Applied Mechanics, v.3, p.61-107, 1953.

GARDINER, C.W. Handbook of Stochastic Methods for Physics, Chemistry and the Natural Sciences. Second Edition - Springer, 1985.

HANNA, S.R. Confidence limits for air quality model evaluations, as estimed by Bootstrap and Jackknife Resampling Methods. Atmospheric Environment, v.23, p.1385-1398, 1989.

MANGIA, C.; MOREIRA, D. M.; SCHIPA, I.; DEGRAZIA, G. A.; TIRABASSI, T.; RIZZA, U. Evaluation of a new eddy diffusivity parameterization from turbulent Eulerian spectra in different stability conditions. Atmospheric Environment, v.36, p.67-76, 2002.

MURGATROYD, R.J. Estimations from geostrophic trajectories of horizontal diffusivity in the mid-latitude troposphere and lower stratosphere. Quarterly Journal of the Royal Meteorological Society, v.95, p.40-62, 1969.

OETTL, D.; ALMBAUER, R.A.; STURM, P.J. A new method to estimate diffusion in stable, low-wind conditions. Journal of Applied Meteorology, v. 40, p. 259-268, 2001.

OETTL, D.; GOULART, A.; DEGRAZIA, G.; ANFOSSI, D. A new hypothesis on meandering atmospheric flows in low wind speed conditions. Atmospheric Environment, v.39, p.1739-1748, 2005.

RODEAN, H.C. Stochastic Lagrangian Models of Turbulence Diffusion. Boston: American Meteorological Society, 84p., 1996.

SAGENDORF, J.F.; DICKSON, C.R. Diffusion under low wind speed, inversion conditions. NOAA Technical Memorandum ERL ARL-52, 89p., 1974.

SHARAN, M.; YADAV, K. Simulation of diffusion experiments under light wind, stable conditions by a variable k-theory model. Atmospheric Environment, v. 32, p. 3481-3492, 1998.

SHARAN, M.; YADAV, A.K.; SING, M.P. Comparison of sigma schemes for estimation of air pollutant dispersion in low winds. Atmospheric Environment, v.29, p.2051-2059, 1995.

THOMSON, D.J. Criteria for the selection of stochastic models of particle trajectories in turbulent flows. Journal of Fluids 
Mechanics, v.180, p.529-556, 1987.

VENKATRAM, A.; WYNGAARD, J.C. Air Pollution Modeling. American Meteorological Society, Boston, 1988.

ZANNETTI, P. Air Pollution Modeling. Teories, Computational Methods and Available Software. Nova York: Kluwer Academic Publisher, 444p., 1990.
ZILITINKEVICH, S.S. On the determination of the height of the Ekman boundary layer. Boundary-Layer Meteorology, v.3, p.141-145, 1972. 